\title{
Discrete Time Semi-Markov Model of a Two Non-Identical Unit Cold Standby System with Preventive Maintenance with Three Modes
}

\author{
Medhat Ahmed El-Damcese ${ }^{1}$, Naglaa Hassan El-Sodany ${ }^{2}$ \\ ${ }^{1}$ Mathematics Department, Faculty of Science, Tanta University, Tanta, Egypt \\ ${ }^{2}$ National Accounts Department, Central Agency for Public Mobilization and Statistics, Cairo, Egypt \\ Email address: \\ meldamcese@yahoo.com (M. A. El-Damcese),naglaa_hassan17@yahoo.com (N. H. El-Sodany)
}

To cite this article:

Medhat Ahmed El-Damcese, Naglaa Hassan El-Sodany. Discrete Time Semi-Markov Model of a Two Non-Identical Unit Cold Standby System with Preventive Maintenance with Three Modes. American Journal of Theoretical and Applied Statistics.

Vol. 4, No. 4, 2015, pp. 277-290. doi: 10.11648/j.ajtas.20150404.18

\begin{abstract}
This paper presents the reliability and availability measures of a two non-identical unit cold standby redundant system (unit-1 is operating, and unit-2 is cold standby) using semi-Markov process under discrete parametric Markov-Chain i.e. failure and repair times of a unit and time to PM and PM time are taken as discrete random variables assuming three different modes (normal $(\mathrm{N})$ mode, partial failure $(\mathrm{P})$ mode and total failure $(\mathrm{F})$ mode) of each unit. The unit-1 is sent for preventive maintenance (PM) after its working for a random period of time assuming that the failure and repair times of a unit and time to PM and PM time are taken as discrete random variables having geometric distributions with different parameters. A single repairman is available with the system for PM of unit-1 and repair of both units. The system is considered in up-state if only one or two units are operative or in partial failure $(\mathrm{P})$ mode. After some basic definitions and notations, we obtain various measures of system effectiveness; reliability, availability, mean time to failure, busy period of repairman due to PM of unit-1, busy period of repairman due to repair of unit-1 and unit-2 from total failure, and the expected profit function using regenerative point technique. The mathematical problem thus developed has next been solved numerically and graphically represented by the aid of Maple program.
\end{abstract}

Keywords: Semi-Markov, Discrete-Time, Cold Standby, Reliability, Mean Sojourn Time, Regenerative Point Technique

\section{Introduction}

Many reliability systems can be modeled using semiMarkov process. The idea of a semi-Markov process was proposed by [1].The essential developments of semi-Markov processes theory applications in reliability were proposed by many authors. [2] and [3] presented a comprehensive treatment of semi-Markov processes and their applications to reliability theory. Some concepts of a semi-Markov process theory was presented by [4]. [5] discussed the basic definitions and theorems from the semi-Markov processes theory and considered the semi-Markov model of the cold standby system with repair. [6] considered the semi-Markov model of multistate system. [7] presented the properties of the reliability function of an object with failure rate modeled by a semi-Markov process applying the renewal equations and obtained the Laplace-Stieltjes transform of the reliability function and its mean time to failure. [8], [9] and [10] studied the semi-Markov processes and their applications in reliability.

In this paper, we are interested in the reliability and availability analysis of a two non-identical unit cold standby system with three modes (normal $(\mathrm{N})$ mode, partial failure $(\mathrm{P})$ mode and total failure (F) mode) with preventive maintenance based on discrete-time semi-Markov processes. In cold standby systems, only one component will be working at any given time, the others being standbys and not working. One of the standby components starts working only when the currently working component fails. Standby components do not fail when they are in standby. The total failure of the primary unit results in the cold standby unit being an operative unit and its failure rate becoming nonzero. The system works until all of its components fail.

Two-unit cold standby redundant system models have been 
analyzed widely in the literature of reliability by many authors. Many authors have analyzed the two-unit cold standby redundant systems with two modes (normal and total failure). [11] proposed a profit analysis of a two non-identical units (unit-1 and unit-2) cold standby system model assuming two modes of each unit. The unit-1 is sent for preventive maintenance (PM) after its working for a random period of time. A single repairman is available with the system for PM of unit-1 and repair of both units. [12] have analyzed the reliability of two-unit cold standby system with single repair. For achieving high reliability of the system, the operative and the standby units are interchanged at random epochs and additional preventive maintenance of operative and the standby unit also. [13] analyzed reliability characteristics of two different series system configurations with mixed standby (include cold and warm standby) components. [14] analyzed the cost analysis of a two dissimilar unit cold standby redundant system subject to inspection and random change in units. In this system each unit works in two different modes normal and total failure. Assuming that the failure, repair, post repair, interchange of units and inspection times are stochastically independent random variables each having an arbitrary distribution. [15] proposed a stochastic behavior of a two-identical unit cold standby system model assuming the two modes of a unit. Two repairmen are considered to repair a failed unit. One is regular repairman and the other is skilled repairman. [16] investigated the stochastic model of a two identical unit cold standby system. If repair of the failed unit is not completed within a specified time, then an order is placed to replace the failed unit by the new one. [17] proposed the stochastic analysis of a single server two identical unit cold standby system. The service facility is summoned whenever the operating unit fails. Many authors have analyzed the two-unit cold standby redundant systems with three modes (normal, partial failure and total failure). [18] studied the two-unit cold standby system with three modes, and the failure and repair times have bivariate exponential density as their joint distribution. [19] have examined a two-unit cold standby system with three modes. The operative unit can fail totally either directly from normal state or via partial failure. [20] studied the behavior of two models with two-unit cold standby system under the assumption that model 1 works in three different modes, but model 2 works in two different modes (Normal and Total failure). [21] studied the stochastic behavior of a twoidentical unit cold standby system model using regenerative point technique assuming three modes of the units where the totally failed unit needs some preparation before going into repair. [22] analyzed a two-identical unit deteriorating standby system using regenerative point technique. Each unit can be in one of the three modes. A unit can fail totally but not partially during its standby state. [23] studied the effect of imperfect switching on a two-unit cold standby system in which each unit works in three different modes. [24] discussed the two-dissimilar-unit cold standby system with preventive maintenance with three modes using linear first order differential equations. [25] studied the availability of the two-identical unit cold standby system with constant failure rates. Initially one unit is operative while the other remains standby. Each of the units of the system has three modes (Normal, Partial failure and Total failure).

Preventive maintenance (PM) is defined as the practice of replacing components or subsystems before they fail in order to promote continuous system operation. PM is also defined as the planned maintenance of components in order to prevent or minimize the breakdowns and the depreciation rates. PM is a sort of repair that is done before the unit actually fails, i.e. after working for a random period of time, a unit goes for its PM. Many authors have analyzed system models with the concept of PM. A wide and recent study of preventive maintenance models was analyzed by [26]. [27] analyzed a series-parallel system with preventive maintenance using semi-Markov process.

The following reliability measures of the system are obtained using regenerative point technique:

i. Transition probabilities and mean sojourn times in various states.

ii. Reliability and mean time to system failure.

iii.Availability and steady state availability of the system.

iv. Expected busy period of the repairman during time interval $(0, t)$

This paper is organized as follows: Section 2 is devoted to the description and basic assumptions of the two-unit cold standby system with preventive maintenance with three modes (normal $(\mathrm{N})$ mode, partial failure $(\mathrm{P})$ mode and total failure (F) mode). Section 3 is devoted to notations and states of the system. Section 4 is devoted to the explanation of transitions between the states. The transition probabilities of the system are given in section 5. Mean sojourn times are given in Section 6. Various measures of system effectiveness; reliability and availability are given in Section 7. In Section 8 we obtained the reliability, availability, steady state availability, mean time to failure of the system and busy period of repairman. The expected profit function of the system is presented in Section 9. In Section 10, a numerical example is given. In Section 11, some concluding remarks are given.

\section{Model Description and Assumptions}

In this paper we consider a reparable discrete time semiMarkov model of a two-unit cold standby system with preventive maintenance, the failure and repair times of a unit and time to PM and PM time are taken as discrete random variables having geometric distributions with different parameters, and we obtain closed form solutions for reliability measures: reliability, availability, steady state availability, mean sojourn time, mean time to failure and busy period of repairman. Regenerative point technique is used for the analysis. Regenerative stochastic process was defined by [1]. The regenerative process is a stochastic process with time points at which, from a probabilistic point of view, the process that restarts itself. These time points are called regenerative points and the state in which regenerative points occur is known as regenerative state.

The following assumptions and notations are associated with the model:

i. The system comprises of two non-identical units 
(unit-1 is operative and unit-2 is cold standby). The system initially starts from state $S_{0}$ where unit- 1 is operative and unit-2 is in cold standby which can't fail in its standby state.

ii. Each unit of the system has three modes: normal $(\mathrm{N})$ mode which means the functioning of the unit with full capacity, partial failure $(\mathrm{P})$ mode which means the functioning of the unit with reduced capacity at a specified level, and total failure (F) mode its capacity goes below specified level.

iii. A unit in the normal $(\mathrm{N})$ mode must pass through the partial failure $(\mathrm{P})$ mode to go to total failure $(\mathrm{F})$ mode.

iv. When the operative unit (unit-1) fails totally from the partial failure $(\mathrm{P})$ mode, the cold standby unit (unit-2) becomes operative.

v. A unit which is repaired in total failure mode go directly to the normal mode without passing through the partial failure mode.

vi. Unit-1 is sent for PM after its working for a random period of time. During the PM action, the unit-1 remains inactive and the system operates with the cold standby unit (unit-2). After completion of PM, unit-1 again starts operation and unit-2 goes into standby.

vii. Single repairman is available with the system to play the role of PM of the operative unit (unit-1) and repair of all units. The unit-1 gets priority in PM and repair over the repair of unit-2.

viii. When unit-1 is in partially failure mode, the installation of the cold standby unit for operation is not permitted.

ix. The unit works as new after preventive maintenance and repair.

x. If unit-1 partially fails at the same epoch when PM of unit-1 is due, then unit-1 enters to partial failure mode and is operative.

xi. All the random variables denoting PM time and time to PM of unit-1, failure times and repair times of both units are independent of discrete nature and follow geometric distributions with different parameters.

xii. The system can perform its purpose even if one unit is in partial failure $(\mathrm{P})$ mode and the other unit is in normal $(\mathrm{N})$ mode or partial failure $(\mathrm{P})$ mode or total failure $(\mathrm{F})$ mode.

xiii. The system remains down when unit-1 is under PM and unit-2 is totally failed.

xiv. The system failure occurs when both units are in total failure (F) mode.

\section{Notations and States of the System}

\subsection{Notations}

$S_{i}:$ state of the $\operatorname{system}(i=0,1,2, \ldots ., 11)$

$E:$ set of regenerative states $\left(S_{0}-S_{8}, S_{10}, S_{11}\right)$ $q_{i, j}(t) / Q_{i, j}(t):$ p.m.f/c.d.f of direct transition time from state $S_{i}$ to state $S_{j}$ during time interval $(0, t)$.

$p_{i, j}$ : steady state transition probability from state $S_{i}$ to state $S_{j}$ during time interval $(0, t)$

$$
p_{i, j}=\lim _{t \rightarrow \infty} Q_{i, j}(t)
$$

$\mu_{i}$ : mean sojourn time in state $S_{i}$

$\alpha_{i} \theta_{i}^{t}:$ p.m.f of partial failure time of unit- $i$

$$
(i=1,2)\left(\alpha_{i}+\theta_{i}=1\right)
$$

$\lambda_{i} \gamma_{i}^{t}:$ p.m.f of total failure time of unit- $i$

$$
(i=1,2)\left(\lambda_{i}+\gamma_{i}=1\right)
$$

$\psi_{i} \sigma_{i}^{t}:$ p.m.f of repair time of unit- $i$ from total failure

$$
(i=1,2)\left(\psi_{i}+\sigma_{i}=1\right)
$$

$\beta_{i} \pi_{i}^{t}:$ p.m.f of repair time of unit- $i$ from partial failure

$$
(i=1,2)\left(\beta_{i}+\pi_{i}=1\right)
$$

$a b^{t}:$ p.m.f of time to PM of unit-1 $(a+b=1)$

$c d^{t}:$ p.m.f of PM time of unit-1 $(c+d=1)$

(C) : symbol used for Laplace convolution

e.g. Let $f(t)$ and $g(t)$ be two non negative discrete functions then the Laplace convolution of the two functions $f(t)$ and $g(t)$ is

$$
\sum_{u=0}^{t} f(u) g(t-u)=f(t) \subseteq g(t-u)
$$

* : symbol used in geometric transformation

$h$ : dummy variable used in geometric transformation

\subsection{Symbols for the States of the Systems}

$N_{i o}$ : Unit- $i(i=1,2)$ in normal $(\mathrm{N})$ mode and operative.

$N_{i s}$ : Unit- $i(i=1,2)$ in normal $(\mathrm{N})$ mode and standby.

$F_{i r}$ : Unit- $i(i=1,2)$ in total failure (F) mode and under repair.

$F_{i w}$ : Unit- $i(i=1,2)$ in total failure (F) mode and waiting for repair.

$P_{i o}$ : Unit- $i(i=1,2)$ in partial failure (P) mode and operative.

$N_{1 p m}$ : Unit-1 in normal (N) mode and under PM.

\subsection{State Specification of the System}

With the help of above symbols the possible states of the 
system are given in Table1.

Table 1. State Specification of the System.

\begin{tabular}{ll}
\hline State & State description \\
\hline$S_{0}:\left(N_{1 o}, N_{2 s}\right)$ & Unit-1 in normal (N) mode and operative, and unit-2 in normal (N) mode and standby \\
$S_{1}:\left(N_{1 p m}, N_{2 o}\right)$ & Uint-1 in normal (N) mode and under PM, and unit-2 in normal (N) mode and operative \\
$S_{2}:\left(N_{1 p m}, P_{2 o}\right)$ & Unit-1 in normal (N) mode and under PM, and unit-2 in partial failure (P) mode and operative \\
$S_{3}:\left(N_{1 o}, F_{2 r}\right)$ & Unit-1 in normal (N) mode and operative, and unit-2 in total failure (F) mode and under repair \\
$S_{4}:\left(P_{1 o}, N_{2 s}\right)$ & Unit-1 in partial failure (P) mode and operative, and unit-2 in normal (N) mode and standby \\
$S_{5}:\left(F_{1 r}, N_{2 o}\right)$ & Unit-1 in total failure (F) mode and under repair, and unit-2 in normal (N) mode and operative \\
$S_{6}:\left(P_{1 o}, F_{2 r}\right)$ & Unit-1 in partial failure (P) mode and operative, and unit-2 in total failure (F) mode and under repair \\
$S_{7}:\left(N_{1 p m}, F_{2 w}\right)$ & Unit-1 in normal (N) mode and under PM, and unit-2 in total failure (F) mode and under waiting for repair \\
$S_{8}:\left(F_{1 r}, P_{2 o}\right)$ & Unit-1 in total failure (F) mode and under repair, and unit-2 in partial failure (P) mode and operative \\
$S_{9}:\left(F_{1 r}, F_{2 w}\right)$ & Unit-1 in total failure (F) mode and under repair, and unit-2 in total failure (F) mode and under waiting for \\
$S_{10}:\left(N_{1 P m}, P_{2 o}\right)$ & repair \\
$S_{11}:\left(P_{1 o}, P_{2 o}\right)$ & Unit-1 in normal (N) mode and under PM, and unit-2 in partial failure (P) mode and operative \\
\hline
\end{tabular}

With the help of above notations and the possible states of the system; the state transition diagram of the two non-identical unit cold standby system is shown in Fig. 1 . The epochs of entry into states $S_{0}, S_{1}, S_{2}, S_{3}, S_{4}, S_{5}, S_{6}, S_{7}, S_{8}, S_{10}$ and $S_{11}$ are regenerative points and thus these states are regenerative states.
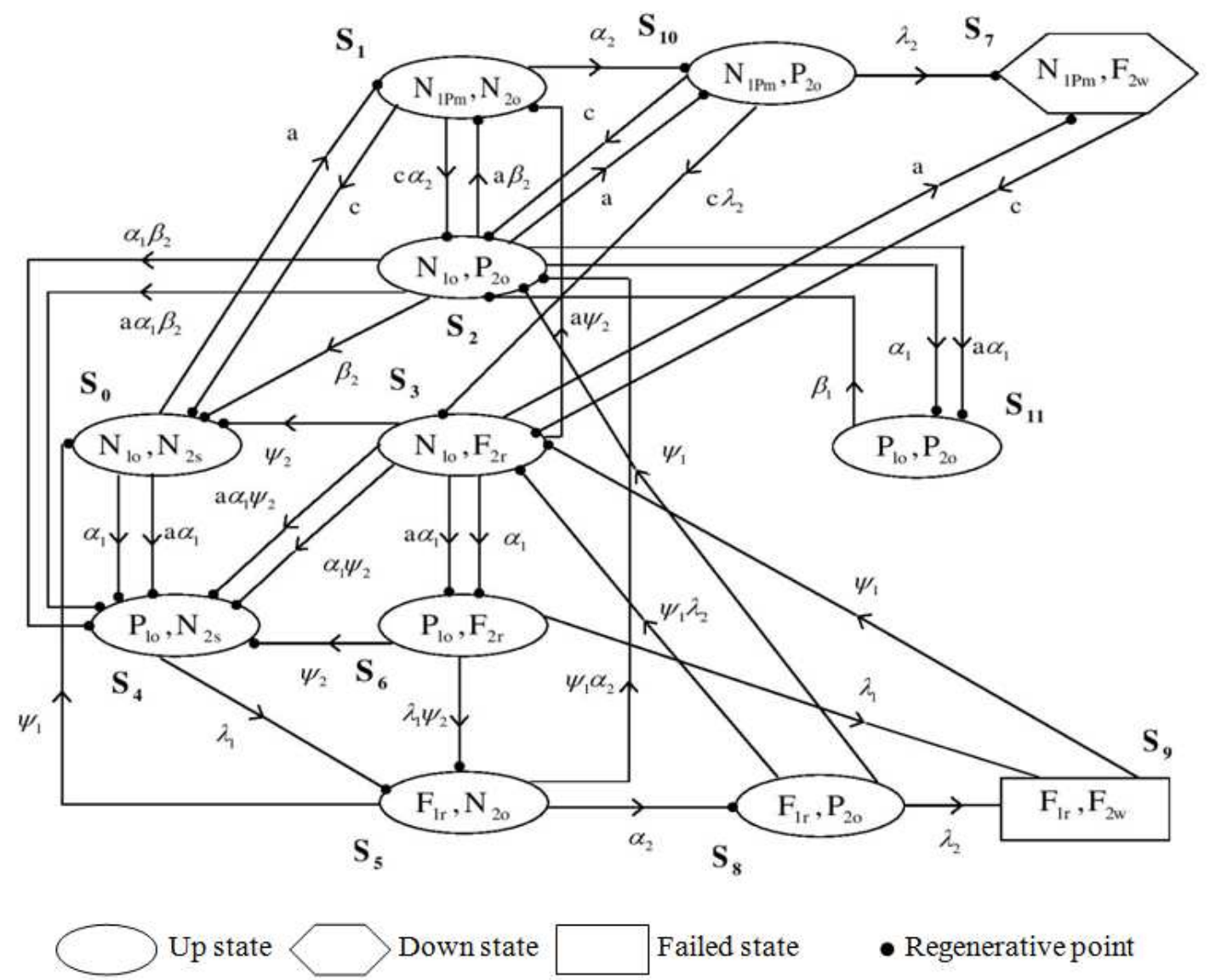

Down state

- Regenerative point

Figure 1. State transition diagram.

Possible states of the system are:

Regenerative states: $S_{0}, S_{1}, S_{2}, S_{3}, S_{4}, S_{5}, S_{6}, S_{7}, S_{8}, S_{10}, S_{11}$

Non-regenerative states: $S_{9}$ 


\section{Explanation of Transitions between the States}

(i) System initially starts from state $S_{0}$ where unit-1 is operative and unit-2 is kept into cold standby which can't fail in its standby state. From this state the system passes to state $S_{4}$ in two mutually exclusive ways:

- Either the operative unit-1 partially fails with rate $\alpha_{1}$ and the standby unit-2 is kept into cold standby.

- The unit-1 partially fails at the same epoch when PM of unit-1 is due. In this case again the situation of state $S_{4}$ arises where the unit-1 partially fails and unit-2 is kept into cold standby.

(ii) The system may transit from state $S_{0}$ to state $S_{1}$ if before the partial failure of unit-1, the PM of unit-1 is due, so that unit-1 enters into PM and unit-2 starts functioning.

(iii) The system transit from state $S_{4}$ to state $S_{5}$ when the partially failed unit-1 fails totally with rate $\lambda_{1}$ and the standby unit-2 becomes operative.

(iv) The system transit from state $S_{5}$ to state $S_{0}$ if before the failure of unit-2, the repair of unit-1 is completed with repair rate $\psi_{1}$.

(v) The system transit from state $S_{5}$ to state $S_{8}$ if before completion of repair of unit-1, unit-2 is partially fails with failure rate $\alpha_{2}$.

(vi) The system transit from state $S_{5}$ to state $S_{2}$ if at the same epoch the repair of unit-1 is completed with repair rate $\psi_{1}$, unit-2 partially fails with failure rate $\alpha_{2}$.

On the same way the transitions between the other states can be observed.

\section{Transition Probabilities of the System}

Let $Q_{i, j}(t)$ be the probability that the system transits from state $S_{i}$ to state $S_{j}$ during time interval $(0, t)$ $, i, j=0,1,2, \ldots, 11$ i.e., if $T_{i j}$ is the transition time from state $S_{i}$ to $S_{j}$ then $Q_{i, j}(t)=\operatorname{Pr}\left[T_{i j} \leq t\right]$ we have

$$
\begin{gathered}
Q_{0,1}(t)=\frac{a \theta_{1}}{1-b \theta_{1}}\left\{1-\left(b \theta_{1}\right)^{t+1}\right\} \\
Q_{0,4}(t)=\frac{a \alpha_{1}+b \alpha_{1}}{1-b \theta_{1}}\left\{1-\left(b \theta_{1}\right)^{t+1}\right\}
\end{gathered}
$$

$$
\begin{aligned}
& Q_{1,0}(t)=\frac{c \theta_{2}}{1-d \theta_{2}}\left\{1-\left(d \theta_{2}\right)^{t+1}\right\} \\
& Q_{1,2}(t)=\frac{c \alpha_{2}}{1-d \theta_{2}}\left\{1-\left(d \theta_{2}\right)^{t+1}\right\} \\
& Q_{1,10}(t)=\frac{d \alpha_{2}}{1-d \theta_{2}}\left\{1-\left(d \theta_{2}\right)^{t+1}\right\} \\
& Q_{2,0}(t)=\frac{b \theta_{1} \beta_{2}}{1-b \theta_{1} \pi_{2}}\left\{1-\left(b \theta_{1} \pi_{2}\right)^{t+1}\right\} \\
& Q_{2,1}(t)=\frac{a \theta_{1} \beta_{2}}{1-b \theta_{1} \pi_{2}}\left\{1-\left(b \theta_{1} \pi_{2}\right)^{t+1}\right\} \\
& Q_{2,4}(t)=\frac{a \alpha_{1} \beta_{2}+b \alpha_{1} \beta_{2}}{1-b \theta_{1} \pi_{2}}\left\{1-\left(b \theta_{1} \pi_{2}\right)^{t+1}\right\} \\
& Q_{2,10}(t)=\frac{a \theta_{1} \pi_{2}}{1-b \theta_{1} \pi_{2}}\left\{1-\left(b \theta_{1} \pi_{2}\right)^{t+1}\right\} \\
& Q_{2,11}(t)=\frac{a \alpha_{1} \pi_{2}+b \alpha_{1} \pi_{2}}{1-b \theta_{1} \pi_{2}}\left\{1-\left(b \theta_{1} \pi_{2}\right)^{t+1}\right\} \\
& Q_{3,0}(t)=\frac{b \psi_{2} \theta_{1}}{1-b \sigma_{2} \theta_{1}}\left\{1-\left(b \sigma_{2} \theta_{1}\right)^{t+1}\right\} \\
& Q_{3,1}(t)=\frac{a \psi_{2} \theta_{1}}{1-b \sigma_{2} \theta_{1}}\left\{1-\left(b \sigma_{2} \theta_{1}\right)^{t+1}\right\} \\
& Q_{3,4}(t)=\frac{a \psi_{2} \alpha_{1}+b \psi_{2} \alpha_{1}}{1-b \sigma_{2} \theta_{1}}\left\{1-\left(b \sigma_{2} \theta_{1}\right)^{t+1}\right\} \\
& Q_{3,6}(t)=\frac{a \sigma_{2} \alpha_{1}+b \sigma_{2} \alpha_{1}}{1-b \sigma_{2} \theta_{1}}\left\{1-\left(b \sigma_{2} \theta_{1}\right)^{t+1}\right\} \\
& Q_{3,7}(t)=\frac{a \sigma_{2} \theta_{1}}{1-b \sigma_{2} \theta_{1}}\left\{1-\left(b \sigma_{2} \theta_{1}\right)^{t+1}\right\} \\
& Q_{4,5}(t)=1-\gamma_{1}^{t+1} \\
& Q_{5,0}(t)=\frac{\psi_{1} \theta_{2}}{1-\sigma_{1} \theta_{2}}\left\{1-\left(\sigma_{1} \theta_{2}\right)^{t+1}\right\} \\
& Q_{5,2}(t)=\frac{\psi_{1} \alpha_{2}}{1-\sigma_{1} \theta_{2}}\left\{1-\left(\sigma_{1} \theta_{2}\right)^{t+1}\right\} \\
& Q_{5,8}(t)=\frac{\sigma_{1} \alpha_{2}}{1-\sigma_{1} \theta_{2}}\left\{1-\left(\sigma_{1} \theta_{2}\right)^{t+1}\right\} \\
& Q_{6,4}(t)=\frac{\gamma_{1} \psi_{2}}{1-\gamma_{1} \sigma_{2}}\left\{1-\left(\gamma_{1} \sigma_{2}\right)^{t+1}\right\}
\end{aligned}
$$




$$
\begin{aligned}
& Q_{6,5}(t)=\frac{\lambda_{1} \psi_{2}}{1-\gamma_{1} \sigma_{2}}\left\{1-\left(\gamma_{1} \sigma_{2}\right)^{t+1}\right\} \\
& Q_{6,9}(t)=\frac{\lambda_{1} \sigma_{2}}{1-\gamma_{1} \sigma_{2}}\left\{1-\left(\gamma_{1} \sigma_{2}\right)^{t+1}\right\} \\
& Q_{7,3}(t)=1-d^{t+1} \\
& Q_{8,2}(t)= \frac{\gamma_{2} \psi_{1}}{1-\gamma_{2} \sigma_{1}}\left\{1-\left(\gamma_{2} \sigma_{1}\right)^{t+1}\right\} \\
& Q_{8,3}(t)= \frac{\lambda_{2} \psi_{1}}{1-\gamma_{2} \sigma_{1}}\left\{1-\left(\gamma_{2} \sigma_{1}\right)^{t+1}\right\} \\
& Q_{8,9}(t)= \frac{\lambda_{2} \sigma_{1}}{1-\gamma_{2} \sigma_{1}}\left\{1-\left(\gamma_{2} \sigma_{1}\right)^{t+1}\right\} \\
& Q_{9,3}(t)=1-\sigma_{1}^{t+1} \\
& Q_{10,2}(t)=\frac{c \gamma_{2}}{1-d \gamma_{2}}\left\{1-\left(d \gamma_{2}\right)^{t+1}\right\} \\
& Q_{10,3}(t)=\frac{c \lambda_{2}}{1-d \gamma_{2}}\left\{1-\left(d \gamma_{2}\right)^{t+1}\right\} \\
& Q_{10,7}(t)=\frac{d \lambda_{2}}{1-d \gamma_{2}}\left\{1-\left(d \gamma_{2}\right)^{t+1}\right\} \\
& Q_{11,2}(t)=1-\pi_{1}^{t+1}
\end{aligned}
$$

$$
\begin{gathered}
p_{2,4}=\frac{a \alpha_{1} \beta_{2}+b \alpha_{1} \beta_{2}}{1-b \theta_{1} \pi_{2}} \\
p_{2,10}=\frac{a \theta_{1} \pi_{2}}{1-b \theta_{1} \pi_{2}} \\
p_{2,11}=\frac{a \alpha_{1} \pi_{2}+b \alpha_{1} \pi_{2}}{1-b \theta_{1} \pi_{2}} \\
p_{3,0}=\frac{b \psi_{2} \theta_{1}}{1-b \sigma_{2} \theta_{1}} \\
p_{3,1}=\frac{a \psi_{2} \theta_{1}}{1-b \sigma_{2} \theta_{1}} \\
p_{8,9}(t)=\frac{a \psi_{2} \alpha_{1}+b \psi_{2} \alpha_{1}}{1-b \sigma_{2} \theta_{1}} \\
p_{8,3}=\frac{\lambda_{2} \sigma_{1}}{1-\gamma_{2} \sigma_{1}}, \\
p_{6,9}=\frac{a \gamma_{2} \alpha_{1}+b \sigma_{2} \alpha_{1}}{1-b \sigma_{2} \theta_{1}} \\
p_{7,3} \sigma_{1,3}=\frac{\lambda_{1} \sigma_{2}}{1-\gamma_{1} \sigma_{2}} \\
p_{3,7}=\frac{a \sigma_{2} \theta_{1}}{1-b \sigma_{2} \theta_{1}} \\
p_{5,2}=\frac{\gamma_{1} \sigma_{1} \alpha_{2}}{1-\sigma_{1} \theta_{2}} \\
p_{5,5}=\frac{\gamma_{1} \psi_{2}}{1-\gamma_{1} \sigma_{2}} \\
1-\sigma_{1} \theta_{2}
\end{gathered}
$$$$
p_{1,0}=\frac{c \theta_{2}}{1-d \theta_{2}}
$$$$
p_{1,2}=\frac{c \alpha_{2}}{1-d \theta_{2}}
$$$$
p_{1,10}=\frac{d \alpha_{2}}{1-d \theta_{2}}
$$$$
p_{2,0}=\frac{b \theta_{1} \beta_{2}}{1-b \theta_{1} \pi_{2}}
$$$$
p_{2,1}=\frac{a \theta_{1} \beta_{2}}{1-b \theta_{1} \pi_{2}}
$$ 


$$
\begin{gathered}
p_{9,3}(t)=1 \\
p_{10,2}=\frac{c \gamma_{2}}{1-d \gamma_{2}} \\
p_{10,3}=\frac{c \lambda_{2}}{1-d \gamma_{2}} \\
p_{10,7}=\frac{d \lambda_{2}}{1-d \gamma_{2}} \\
p_{11,2}=1
\end{gathered}
$$

We observe that the following relations hold:

$$
\begin{gathered}
p_{0,1}+p_{0,4}=1 \\
p_{1,0}+p_{1,2}+p_{1,10}=1 \\
p_{2,0}+p_{2,1}+p_{2,4}+p_{2,10}+p_{2,11}=1 \\
p_{3,0}+p_{3,1}+p_{3,4}+p_{3,6}+p_{3,7}=1 \\
p_{4,5}=p_{7,3}=p_{9,3}=p_{11,2}=1 \\
p_{5,0}+p_{5,2}+p_{5,8}=1 \\
p_{6,4}+p_{6,5}+p_{6,9}=1 \\
p_{8,2}+p_{8,3}+p_{8,9}=1 \\
p_{10,2}+p_{10,3}+p_{10,7}=1
\end{gathered}
$$

\section{Mean Sojourn Times}

The mean sojourn time $\mu_{i}$ in state $S_{i}$ is defined as the time spent in state $S_{i}$ before transiting to any other state.

Let $T_{i}$ be the time spent in state $S_{i}$. Then the mean sojourn time in states $S_{i}$ can be obtained as follows:

$$
\mu_{i}=E\left(T_{i}\right)=\sum_{t=0}^{\infty} P\left(T_{i}>t\right) \quad, i=0,1,2, \ldots, 11
$$

Hence

$$
\begin{gathered}
\mu_{0}=\frac{b \theta_{1}}{1-b \theta_{1}} \\
\mu_{1}=\frac{d \theta_{2}}{1-d \theta_{2}} \\
\mu_{2}=\frac{b \theta_{1} \pi_{2}}{1-b \theta_{1} \pi_{2}}
\end{gathered}
$$

$$
\begin{gathered}
\mu_{3}=\frac{b \sigma_{2} \theta_{1}}{1-b \sigma_{2} \theta_{1}} \\
\mu_{4}=\frac{\gamma_{1}}{1-\gamma_{1}} \\
\mu_{5}=\frac{\sigma_{1} \theta_{2}}{1-\sigma_{1} \theta_{2}} \\
\mu_{6}=\frac{\gamma_{1} \sigma_{2}}{1-\gamma_{1} \sigma_{2}} \\
\mu_{7}=\frac{d}{1-d} \\
\mu_{8}=\frac{\gamma_{2} \sigma_{1}}{1-\gamma_{2} \sigma_{1}} \\
\mu_{9}=\frac{\psi_{1}}{1-\psi_{1}} \\
\mu_{10}=\frac{d \gamma_{2}}{1-d \gamma_{2}} \\
\pi_{1}
\end{gathered}
$$

\section{Measures of System Effectiveness}

In order to obtain various interesting measures of system effectiveness we develop the following relations for reliability, availability, steady state availability, mean time to failure and expected busy period of the repairman during time interval $(0, t)$ :

\subsection{Reliability of the System}

The reliability function $R_{i}(t)$ is the probability that a system will be successfully operating without failure in the interval from time 0 to time $t$ when it initially starts from operative up state $S_{i} ; i=0,1, \ldots, 8,10,11$. To determine it, we regard the failed state $S_{9}$ as absorbing state. Now we have the following set of convolution equations for $R_{i}(t) ; i=0,1, \ldots ., 8,10,11$

$$
\begin{aligned}
R_{i}(t) & =Z_{i}(t)+\sum_{j}\left(\sum_{u=0}^{t-1} q_{i j}(u) R_{j}(t-1-u)\right) \\
& =Z_{i}(t)+\sum_{j} q_{i j}(t-1) \subset R_{j}(t-1)
\end{aligned}
$$

where $j$ is any successive state to which state $i$ can transit. where,

$$
Z_{0}(t)=b^{t} \theta_{1}^{t}
$$




$$
\begin{gathered}
Z_{1}(t)=d^{t} \theta_{2}^{t} \\
Z_{2}(t)=b^{t} \theta_{1}^{t} \pi_{2}^{t} \\
Z_{3}(t)=b^{t} \sigma_{2}^{t} \theta_{1}^{t} \\
Z_{4}(t)=\gamma_{1}^{t} \\
Z_{5}(t)=\sigma_{1}^{t} \theta_{2}^{t} \\
Z_{6}(t)=\gamma_{1}^{t} \sigma_{2}^{t} \\
Z_{7}(t)=d^{t} \\
Z_{8}(t)=\gamma_{2}^{t} \sigma_{1}^{t} \\
Z_{10}(t)=d^{t} \gamma_{2}^{t} \\
Z_{11}(t)=\pi_{1}^{t}
\end{gathered}
$$

Then

$$
\begin{aligned}
& R_{0}(t)=Z_{0}(t)+q_{0,1}(t-1) \Subset R_{1}(t-1)+q_{0,4}(t-1) \Subset R_{4}(t-1) \\
& R_{1}(t)=Z_{1}(t)+q_{1,0}(t-1) \subseteq R_{0}(t-1) \\
& +q_{1,2}(t-1) \subseteq R_{2}(t-1)+q_{1,10}(t-1) \subseteq R_{10}(t-1) \\
& R_{2}(t)=Z_{2}(t)+q_{2,0}(t-1) \odot R_{0}(t-1) \\
& +q_{2,1}(t-1) \odot R_{1}(t-1)+q_{2,4}(t-1) \oplus R_{4}(t-1) \\
& +q_{2,10}(t-1) \subseteq R_{10}(t-1)+q_{2,11}(t-1) \subseteq R_{11}(t-1) \\
& R_{3}(t)=Z_{3}(t)+q_{3,0}(t-1) \subseteq R_{0}(t-1) \\
& +q_{3,1}(t-1) \oplus R_{1}(t-1)+q_{3,4}(t-1) \odot R_{4}(t-1) \\
& +q_{3,6}(t-1) \oplus R_{6}(t-1)+q_{3,7}(t-1) \odot R_{7}(t-1) \\
& R_{4}(t)=Z_{4}(t)+q_{4,5}(t-1) \subset R_{5}(t-1) \\
& R_{5}(t)=Z_{5}(t)+q_{5,0}(t-1) \subseteq R_{0}(t-1) \\
& +q_{5,2}(t-1) @ R_{2}(t-1)+q_{5,8}(t-1) @ R_{8}(t-1) \\
& R_{6}(t)=Z_{6}(t)+q_{6,4}(t-1) \subseteq R_{4}(t-1)+q_{6,5}(t-1) \subseteq R_{5}(t-1) \\
& R_{7}(t)=Z_{7}(t)+q_{7,3}(t-1) \subseteq R_{3}(t-1) \\
& R_{8}(t)=Z_{8}(t)+q_{8,2}(t-1) \Subset R_{2}(t-1)+q_{8,3}(t-1) \oplus R_{3}(t-1) \\
& R_{10}(t)=Z_{10}(t)+q_{10,2}(t-1) \subseteq R_{2}(t-1) \\
& +q_{10,3}(t-1) \subseteq R_{3}(t-1)+q_{10,7}(t-1) \subseteq R_{7}(t-1) \\
& R_{11}(t)=Z_{11}(t)+q_{11,2}(t-1) \Subset R_{2}(t-1)
\end{aligned}
$$

\subsection{Availability of the System}

At time $t=0$ the system start operation with no failed units. The availability function of a system, denoted by $A_{i}(t)$, is defined as the probability that the system is available at time $t$ when it initially starts from state $S_{i} ; i=0,1,2, \ldots, 11$. The following recurrence relations can be easily developed for $A_{i}(t) ; i=0,1,2, \ldots, 11$.

$$
\begin{aligned}
A_{i}(t) & =Z_{i}(t)+\sum_{j}\left(\sum_{u=0}^{t-1} q_{i j}(u) A_{j}(t-1-u)\right) \\
& =Z_{i}(t)+\sum_{j} q_{i j}(t-1) \subset A_{j}(t-1)
\end{aligned}
$$

where $j$ is any successive state to which state $i$ can transit, and $Z_{i}(t) ; i=0,1,2, \ldots ., 8,10,11$ are the same as given in the reliability of the system's section.

Then

$$
\begin{aligned}
& A_{0}(t)=Z_{0}(t)+q_{0,1}(t-1) \Subset A_{1}(t-1)+q_{0,4}(t-1) \Subset A_{4}(t-1) \\
& A_{1}(t)=Z_{1}(t)+q_{1,0}(t-1) \subseteq A_{0}(t-1) \\
& +q_{1,2}(t-1) \subseteq A_{2}(t-1)+q_{1,10}(t-1) \subseteq A_{10}(t-1) \\
& A_{2}(t)=Z_{2}(t)+q_{2,0}(t-1) \subseteq A_{0}(t-1) \\
& +q_{2,1}(t-1) \subseteq A_{1}(t-1)+q_{2,4}(t-1) \subseteq A_{4}(t-1) \\
& +q_{2,10}(t-1) \subset A_{10}(t-1)+q_{2,11}(t-1) \subset A_{11}(t-1) \\
& A_{3}(t)=Z_{3}(t)+q_{3,0}(t-1) \subseteq A_{0}(t-1) \\
& +q_{3,1}(t-1) \subseteq A_{1}(t-1)+q_{3,4}(t-1) \subseteq A_{4}(t-1) \\
& +q_{3,6}(t-1) \Subset A_{6}(t-1)+q_{3,7}(t-1) \Subset A_{7}(t-1) \\
& A_{4}(t)=Z_{4}(t)+q_{4,5}(t-1) \Subset A_{5}(t-1) \\
& A_{5}(t)=Z_{5}(t)+q_{5,0}(t-1) \subseteq A_{0}(t-1) \\
& +q_{5,2}(t-1) \subseteq A_{2}(t-1)+q_{5,8}(t-1) \subseteq A_{8}(t-1) \\
& A_{6}(t)=Z_{6}(t)+q_{6,4}(t-1) \subseteq A_{4}(t-1) \\
& +q_{6,5}(t-1) \subseteq A_{5}(t-1)+q_{6,9}(t-1) \subseteq A_{9}(t-1) \\
& A_{7}(t)=q_{7,3}(t-1) \Subset A_{3}(t-1) \\
& A_{8}(t)=Z_{8}(t)+q_{8,2}(t-1) \oplus A_{2}(t-1) \\
& +q_{8,3}(t-1) \subseteq A_{3}(t-1)+q_{8,9}(t-1) \subseteq A_{9}(t-1) \\
& A_{9}(t)=q_{9,3}(t-1) \subseteq A_{3}(t-1) \\
& A_{10}(t)=Z_{10}(t)+q_{10,2}(t-1) @ A_{2}(t-1) \\
& +q_{10,3}(t-1) \Subset A_{3}(t-1)+q_{10,7}(t-1) \subseteq A_{7}(t-1) \\
& A_{11}(t)=Z_{11}(t)+q_{11,2}(t-1) \Subset A_{2}(t-1)
\end{aligned}
$$




\subsection{Busy Period of Repairman}

\subsubsection{Due to PM of Unit-1}

Let $B_{i}^{p m}(t)$ be the probability that the repairman is busy at epoch $(t-1)$ in the PM of an operative unit-1 when system initially starts from state $S_{i} ; i=0,1,2, \ldots, 11$. Using simple probabilistic arguments as in case of reliability, the recurrence relations for $B_{i}^{p m}(t) ; i=0,1,2, \ldots ., 11$ can be easily developed as below:

$$
\begin{aligned}
& B_{o}^{p m}(t)=q_{0,1}(t-1) \odot B_{1}^{p m}(t-1)+q_{0,4}(t-1) \odot B_{4}^{p m}(t-1) \\
& B_{1}^{p m}(t)=Z_{1}(t)+q_{1,0}(t-1) \odot B_{0}^{p m}(t-1) \\
& +q_{1,2}(t-1) \odot B_{2}^{p m}(t-1)+q_{1,10}(t-1) \odot B_{10}^{p m}(t-1) \\
& B_{2}^{p m}(t)=q_{2,0}(t-1) \odot B_{0}^{p m}(t-1) \\
& +q_{2,1}(t-1) \odot B_{1}^{p m}(t-1)+q_{2,4}(t-1) \odot B_{4}^{p m}(t-1) \\
& +q_{2,10}(t-1) \odot B_{10}^{p m}(t-1)+q_{2,11}(t-1) \odot B_{11}^{p m}(t-1) \\
& B_{3}^{p m}(t)=q_{3,0}(t-1) \odot B_{0}^{p m}(t-1) \\
& +q_{3,1}(t-1) \odot B_{1}^{p m}(t-1)+q_{3,4}(t-1) \odot B_{4}^{p m}(t-1) \\
& +q_{3,6}(t-1) \odot B_{6}^{p m}(t-1)+q_{3,7}(t-1) \odot B_{7}^{p m}(t-1) \\
& B_{4}^{p m}(t)=q_{4,5}(t-1) \odot B_{5}^{p m}(t-1) \\
& B_{5}^{p m}(t)=q_{5,0}(t-1) \odot B_{0}^{p m}(t-1) \\
& +q_{5,2}(t-1) \odot B_{2}^{p m}(t-1)+q_{5,8}(t-1) \odot B_{8}^{p m}(t-1) \\
& B_{6}^{p m}(t)=q_{6,4}(t-1) \odot B_{4}^{p m}(t-1) \\
& +q_{6,5}(t-1) \odot B_{5}^{p m}(t-1)+q_{6,9}(t-1) \odot B_{9}^{p m}(t-1) \\
& B_{7}^{p m}(t)=Z_{7}(t)+q_{7,3}(t-1) \oplus B_{3}^{p m}(t-1) \\
& B_{8}^{p m}(t)=q_{8,2}(t-1) \odot B_{2}^{p m}(t-1) \\
& +q_{8,3}(t-1) \odot B_{3}^{p m}(t-1)+q_{8,9}(t-1) \odot B_{9}^{p m}(t-1) \\
& B_{9}^{p m}(t)=q_{9,3}(t-1) \oplus B_{3}^{p m}(t-1) \\
& B_{10}^{p m}(t)=Z_{10}(t)+q_{10,2}(t-1) \odot B_{2}^{p m}(t-1) \\
& +q_{10,3}(t-1) \odot B_{3}^{p m}(t-1)+q_{10,7}(t-1) \odot B_{7}^{p m}(t-1) \\
& B_{11}^{p m}(t)=q_{11,2}(t-1) \odot B_{2}^{p m}(t-1)
\end{aligned}
$$

where the values of $Z_{1}(t), Z_{7}(t), Z_{10}(t)$ are the same as given in the reliability of the system's section.

\subsubsection{Due to Repair of Unit-1 and Unit-2 from Total Failure}

Let $B_{i}^{r_{1}}(t)$ and $B_{i}^{r_{2}}(t)$ be the respective probabilities that the repairman is busy at epoch $(t-1)$ in the repair of unit-1 and unit-2 from, when system initially starts from state $S_{i}$. Using simple probabilistic arguments as in case of reliability, the recurrence relations for $B_{i}^{j}(t) ; i=0,1,2, \ldots, 11$ can be easily developed as below. The dichotomous variable $\delta$ takes values 1 and 0 respectively for $j=r_{1}$ and $j=r_{2}$.

$$
\begin{aligned}
& B_{o}^{j}(t)=q_{0,1}(t-1) \odot B_{1}^{j}(t-1)+q_{0,4}(t-1) \odot B_{4}^{j}(t-1) \\
& B_{1}^{j}(t)=q_{1,0}(t-1) \oplus B_{0}^{j}(t-1) \\
& +q_{1,2}(t-1) \odot B_{2}^{j}(t-1)+q_{1,10}(t-1) \odot B_{10}^{j}(t-1) \\
& B_{2}^{j}(t)=q_{2,0}(t-1) \odot B_{0}^{j}(t-1)+q_{2,1}(t-1) \odot B_{1}^{j}(t-1) \\
& +q_{2,4}(t-1) \odot B_{4}^{j}(t-1)+q_{2,10}(t-1) \odot B_{10}^{j}(t-1) \\
& +q_{2,11}(t-1) \odot B_{11}^{j}(t-1) \\
& B_{3}^{j}(t)=(1-\delta) Z_{3}(t)+q_{3,0}(t-1) \odot B_{0}^{j}(t-1) \\
& +q_{3,1}(t-1) \odot B_{1}^{j}(t-1)+q_{3,4}(t-1) \odot B_{4}^{j}(t-1) \\
& +q_{3,6}(t-1) \odot B_{6}^{j}(t-1)+q_{3,7}(t-1) \odot B_{7}^{j}(t-1) \\
& B_{4}^{j}(t)=q_{4,5}(t-1) \oplus B_{5}^{j}(t-1) \\
& B_{5}^{j}(t)=\delta Z_{5}(t)+q_{5,0}(t-1) \odot B_{0}^{j}(t-1) \\
& +q_{5,2}(t-1) \odot B_{2}^{j}(t-1)+q_{5,8}(t-1) \odot B_{8}^{j}(t-1) \\
& B_{6}^{j}(t)=(1-\delta) Z_{6}(t)+q_{6,4}(t-1) \odot B_{4}^{j}(t-1) \\
& +q_{6,5}(t-1) \odot B_{5}^{j}(t-1)+q_{6,9}(t-1) \odot B_{9}^{j}(t-1) \\
& B_{7}^{j}(t)=q_{7,3}(t-1) \odot B_{3}^{j}(t-1) \\
& B_{8}^{j}(t)=\delta Z_{8}(t)+q_{8,2}(t-1) \odot B_{2}^{j}(t-1) \\
& +q_{8,3}(t-1) \odot B_{3}^{j}(t-1)+q_{8,9}(t-1) \odot B_{9}^{j}(t-1) \\
& B_{9}^{j}(t)=\delta Z_{9}(t)+q_{9,3}(t-1) \odot B_{3}^{j}(t-1) \\
& B_{10}^{j}(t)=q_{10,2}(t-1) \oplus B_{2}^{j}(t-1) \\
& +q_{10,3}(t-1) \odot B_{3}^{j}(t-1)+q_{10,7}(t-1) \odot B_{7}^{j}(t-1) \\
& B_{11}^{j}(t)=q_{11,2}(t-1) \odot B_{2}^{j}(t-1)
\end{aligned}
$$

where the values of $Z_{i}(t) ; i=3,5,6,8$ are the same as given in the reliability of the system's section, and

$$
Z_{9}(t)=\psi_{1}^{t+1}
$$

\section{Analysis of Characteristics}

\subsection{Reliability and Mean Time to Failure Analysis}

Taking geometric transforms of (75-85), we obtain the following set of algebraic equations: 


$$
\begin{gathered}
R_{0}^{*}(h)=Z_{0}^{*}+h q_{0,1}^{*} R_{1}^{*}(h)+h q_{0,4}^{*} R_{4}^{*}(h) \\
R_{1}^{*}(h)=Z_{1}^{*}+h q_{1,0}^{*} R_{0}^{*}(h)+h q_{1,2}^{*} R_{2}^{*}(h)+h q_{1,10}^{*} R_{10}^{*}(h) \\
R_{2}^{*}(h)=Z_{2}^{*}+h q_{2,0}^{*} R_{0}^{*}(h)+h q_{2,1}^{*} R_{1}^{*}(h) \\
+h q_{2,4}^{*} R_{4}^{*}(h)+h q_{2,10}^{*} R_{10}^{*}(h)+h q_{2,11}^{*} R_{11}^{*}(h) \\
R_{3}^{*}(h)=Z_{3}^{*}+h q_{3,0}^{*} R_{0}^{*}(h)+h q_{3,1}^{*} R_{1}^{*}(h)+h q_{3,4}^{*} R_{4}^{*}(h) \\
+h q_{3,6}^{*} R_{6}^{*}(h)+h q_{3,7}^{*} R_{7}^{*}(h) \\
R_{4}^{*}(h)=Z_{4}^{*}+h q_{4,5}^{*} R_{5}^{*}(h) \\
R_{5}^{*}(h)=Z_{5}+h q_{5,0}^{*} R_{0}^{*}(h)+h q_{5,2}^{*} R_{2}^{*}(h)+h q_{5,8}^{*} R_{8}^{*}(h) \\
R_{6}^{*}(h)=Z_{6}^{*}+h q_{6,4}^{*} R_{4}^{*}(h)+h q_{6,5}^{*} R_{5}^{*}(h) \\
R_{7}^{*}(h)=Z_{7}^{*}+q_{7,3}^{*} R_{3}^{*}(h) \\
R_{8}^{*}(h)=Z_{8}^{*}+h q_{8,2}^{*} R_{2}^{*}(h)+h q_{8,3}^{*} R_{3}^{*}(h) \\
R_{10}^{*}(h)=Z_{10}^{*}+h q_{10,2}^{*} R_{2}^{*}(h)+h q_{10,3}^{*} R_{3}^{*}(h)+h q_{10,7}^{*} R_{7}^{*}(h) \\
R_{11}^{*}(h)=Z_{11}^{*}+q_{11,2}^{*} R_{2}^{*}(h) \quad(122-132)
\end{gathered}
$$

Simplifying (122-132) for $R_{0}^{*}(h)$, but it is difficult to solve (122-132) since expressions for geometric transforms of reliability functions $R_{i}^{*}(h)$ are in very complicated form and the complexity increases with the increase in number of equations. We put it in the following form:

$$
R_{0}^{*}(h)=\frac{N_{1}(h)}{D_{1}(h)}
$$

Collecting the coefficients of $h^{t}$ from (133), we can get the reliability of the system $R_{0}(t)$.

The mean time to system failure is

$$
M T T F=\lim _{h \rightarrow 1} R_{0}^{*}(h)=\frac{N_{1}(1)}{D_{1}(1)}-1
$$

\subsection{Availability Analysis}

Taking geometric transforms of (86-97), we obtain the following set of algebraic equations:

$$
\begin{gathered}
A_{0}^{*}(h)=Z_{0}^{*}+h q_{0,1}^{*} A_{1}^{*}(h)+h q_{0,4}^{*} A_{4}^{*}(h) \\
A_{1}^{*}(h)=Z_{1}^{*}+h q_{1,0}^{*} A_{0}^{*}(h)+h q_{1,2}^{*} A_{2}^{*}(h)+h q_{1,10}^{*} A_{10}^{*}(h) \\
A_{2}(h)=Z_{2}^{*}+h q_{2,0}^{*} A_{0}^{*}(h)+h q_{2,1}^{*} A_{1}^{*}(h)+h q_{2,4}^{*} A_{4}^{*}(h) \\
+h q_{2,10}^{*} A_{10}^{*}(h)+h q_{2,11}^{*} A_{11}^{*}(h)
\end{gathered}
$$

$$
\begin{gathered}
A_{3}^{*}(h)=Z_{3}^{*}+h q_{3,0}^{*} A_{0}^{*}(h)+h q_{3,1}^{*} A_{1}^{*}(h)+h q_{3,4}^{*} A_{4}^{*}(h) \\
+h q_{3,6}^{*} A_{6}(h)+h q_{3,7}^{*} A_{7}^{*}(h) \\
A_{4}^{*}(h)=Z_{4}^{*}+q_{4,5}^{*} A_{5}^{*}(h) \\
A_{5}^{*}(h)=Z_{5}^{*}+h q_{5,0}^{*} A_{0}^{*}(h)+h q_{5,2}^{*} A_{2}(h)+h q_{5,8}^{*} A_{8}^{*}(h) \\
A_{6}^{*}(h)=Z_{6}^{*}+h q_{6,4}^{*} A_{4}^{*}(h)+h q_{6,5}^{*} A_{5}^{*}(h)+h q_{6,9}^{*} A_{9}^{*}(h) \\
A_{7}^{*}(h)=h q_{7,3}^{*} A_{3}^{*}(h) \\
A_{8}^{*}(h)=Z_{8}^{*}+h q_{8,2}^{*} A_{2}^{*}(h)+h q_{8,3}^{*} A_{3}^{*}(h)+h q_{8,9}^{*} A_{9}^{*}(h) \\
A_{9}^{*}(h)=h q_{9,3}^{*} A_{3}^{*}(h) \\
A_{10}^{*}(h)=Z_{10}^{*}+h q_{10,2}^{*} A_{2}^{*}(h)+h q_{10,3}^{*} A_{3}^{*}(h)+h q_{10,7}^{*} A_{7}^{*}(h) \\
A_{11}^{*}(h)=Z_{11}^{*}+h q_{11,2}^{*} A_{2}^{*}(h)
\end{gathered}
$$

Simplifying (135-146)for $A_{0}^{*}(h)$, as in the case of reliability function, we put it in the following form:

$$
A_{0}^{*}(h)=\frac{N_{2}(h)}{D_{2}(h)}
$$

The steady state availability of the system is given by:

$$
A_{0}=\lim _{t \rightarrow \infty} A_{0}(t)=\lim _{h \rightarrow 1}(1-h) \frac{N_{2}(h)}{D_{2}(h)}
$$

As $D_{2}(h)$ at $h=1$ is zero, hence by applying L. Hospital Rule, we get

$$
A_{0}=-\frac{N_{2}(1)}{D_{2}^{\prime}(1)}
$$

Now the expected up time of the system up to epoch $(t-1)$ is given by:

$$
\mu_{u p}(t)=\sum_{x=0}^{t-1} A_{0}(x)
$$

So that

$$
\mu_{u p}^{*}(h)=\frac{A_{0}^{*}(h)}{(1-h)}
$$

\subsection{Busy Period Analysis}

\subsubsection{Due to PM of Unit-1}

Taking geometric transforms of (98-109), we obtain the following set of algebraic equations:

$$
B_{o}^{p m^{*}}(h)=h q_{0,1}^{*} B_{1}^{p m^{*}}(h)+h q_{0,4}^{*} B_{4}^{p m^{*}}(h)
$$




$$
\begin{gathered}
B_{1}^{p m^{*}}(h)=Z_{1}^{*}+h q_{1,0}^{*} B_{0}^{p m^{*}}(h)+h q_{1,2}^{*} B_{2}^{p m^{*}}(h)+h q_{1,10}^{*} B_{10}^{p m^{*}}(h) \\
B_{2}^{p m^{*}}(h)=q_{2,0}^{*} B_{0}^{p m^{*}}(h)+q_{2,1}^{*} B_{1}^{p m^{*}}(h)+q_{2,4}^{*} B_{4}^{p m^{*}}(h) \\
+q_{2,10}^{*} B_{10}^{p m^{*}}(h)+q_{2,11}^{*} B_{11}^{p m^{*}}(h) \\
B_{3}^{p m^{*}}(h)=q_{3,0}^{*} B_{0}^{p m^{*}}(h)+q_{3,1}^{*} B_{1}^{p m^{*}}(h)+q_{3,4}^{*} B_{4}^{p m^{*}}(h) \\
+q_{3,6}^{*} B_{6}^{p m^{*}}(h)+q_{3,7}^{*} B_{7}^{p m m^{*}}(h) \\
B_{4}^{p m^{*}}(t)=h q_{4,5}^{*} B_{5}^{p m^{*}}(h) \\
B_{5}^{p m^{*}}(h)=h q_{5,0}^{*} B_{0}^{p m^{*}}(h)+h q_{5,2}^{*} B_{2}^{p m^{*}}(h)+h q_{5,8}^{*} B_{8}^{p m^{*}}(h) \\
B_{6}^{p m^{*}}(h)=h q_{6,4}^{*} B_{4}^{p m^{*}}(h)+h q_{6,5}^{*} B_{5}^{p m^{*}}(h)+h q_{6,9}^{*} B_{9}^{p m^{*}}(h) \\
B_{7}^{p m^{*}}(t)=Z_{7}^{*}+h q_{7,3}^{*} B_{3}^{p m^{*}}(h) \\
B_{8}^{p m^{*}}(h)=h q_{8,2}^{*} B_{2}^{p m^{*}}(h)+h q_{8,3}^{*} B_{3}^{p m^{*}}(h)+h q_{8,9}^{*} B_{9}^{p m^{*}}(h) \\
B_{9}^{p m^{*}}(h)=h q_{9,3}^{*} B_{3}^{p m^{*}}(h) \\
B_{10}^{p m^{*}}(h)=Z_{10}^{*}+h q_{10,2}^{*} B_{2}^{p m^{*}}(h)+h q_{10,3}^{*} B_{3}^{p m^{*}}(h) \\
+h q_{10,7}^{*} B_{7}^{p m^{*}}(h) \\
B_{11}^{p m^{*}}(h)=h q_{11,2}^{*} B_{2}^{p m^{*}}(h)
\end{gathered}
$$

Simplifying (150-161) for $B_{0}^{* p m}(h)$, we get

$$
B_{0}^{p m^{*}}(h)=\frac{N_{3}(h)}{D_{2}(h)}
$$

where $D_{2}(h)$ is same as in availability analysis.

In the long run, the probability that the repairman is busy in PM of unit-1 is given by:

$$
B_{0}^{p m}=\lim _{t \rightarrow \infty} B_{0}^{p m}(t)=\lim _{h \rightarrow 1}(1-h) \frac{N_{3}(h)}{D_{2}(h)}
$$

But $D_{2}(h)$ at $h=1$ is zero, hence by applying L. Hospital Rule, we get

$$
B_{0}^{p m}=-\frac{N_{3}(1)}{D_{2}^{\prime}(1)}
$$

Now the expected busy period of the repairman in the repair of a failed unit up to epoch $(t-1)$ is given by:

$$
\mu_{b}^{p m}(t)=\sum_{x=0}^{t-1} B_{0}^{p m}(x)
$$

So that

$$
\mu_{b}^{p m^{*}}(h)=\frac{B_{0}^{p m^{*}}(h)}{(1-h)}
$$

\subsubsection{Due to Repair of Unit-1 and Unit-2 from Total}

\section{Failure}

Taking geometric transforms of (110-121), we obtain the following set of algebraic equations:

$$
\begin{gathered}
B_{o}^{j^{*}}(h)=h q_{0,1}^{*} B_{1}^{j^{*}}(h)+h q_{0,4}^{*} B_{4}^{j^{*}}(h) \\
B_{1}^{j^{*}}(h)=q_{1,0} B_{0}^{j^{*}}(h)+q_{1,2} B_{2}^{j^{*}}(h)+h q_{1,10} B_{10}^{j^{*}}(h) \\
B_{2}^{j^{*}}(h)=h q_{2,0}^{*} B_{0}^{j^{*}}(h)+h q_{2,1}^{*} B_{1}^{j 8}(h)+h q_{2,4}^{*} B_{4}^{j^{*}}(h) \\
+h q_{2,10}^{*} B_{10}^{j^{*}}(h)+h q_{2,11}^{*} B_{11}^{j^{*}}(h) \\
B_{3}^{j^{*}}(h)=(1-\delta) Z_{3}^{*}+h q_{3,0}^{*} B_{0}^{j^{*}}(h)+h q_{3,1}^{*} B_{1}^{j^{*}}(h) \\
+h q_{3,4}^{*} B_{4}^{j^{*}}(h)+h q_{3,6}^{*} B_{6}^{j^{*}}(h)+h q_{3,7}^{*} B_{7}^{j^{*}}(h) \\
B_{5}^{j^{*}}(h)=\delta Z_{5}^{*}+h q_{5,0}^{*} B_{0}^{j^{*}}(h)+h q_{5,2}^{*} B_{2}^{j^{*}}(h)+h q_{5,8}^{*} B_{8}^{j^{*}}(h) \\
B_{6}^{j^{*}}(h)=(1-\delta) Z_{6}^{*}+h q_{6,4}^{*} B_{4}^{j^{*}}(h)+h q_{6,5}^{*} B_{5}^{j^{*}}(h) \\
+h q_{6,9}^{*} B_{9}^{j^{*}}(h) \\
B_{7}^{j^{*}}(h)=q_{7,3}^{*} B_{3}^{j^{*}}(h) \\
B_{8}^{j^{*}}(h)=\delta Z_{8}^{*}+h q_{8,2}^{*} B_{2}^{j^{*}}(h)+h q_{8,3}^{*} B_{3}^{j^{*}}(h)+h q_{8,9}^{*} B_{9}^{j^{*}}(h) \\
B_{9}^{j^{*}}(h)=\delta Z_{9}^{*}+h q_{9,3}^{*} B_{3}^{j^{*}}(h) \\
B_{10}^{j^{*}}(t)=h q_{10,2}^{*} B_{2}^{j^{*}}(h)+h q_{10,3}^{*} B_{3}^{j^{*}}(h)+h q_{10,7}^{*} B_{7}^{j^{*}}(h) \\
B_{11}^{j^{*}}(h)=q_{11,2}^{*} B_{2}^{j^{*}}(h)
\end{gathered}
$$

Simplifying (164-175) for $j=r_{1}$ and $j=r_{2}$, we get

$$
\begin{gathered}
B_{0}^{r_{1}^{*} *}(h)=\frac{N_{4}(h)}{D_{2}(h)} \\
B_{0}^{r^{2 *}}(h)=\frac{N_{5}(h)}{D_{2}(h)}
\end{gathered}
$$

where $D_{2}(h)$ is same as in availability analysis.

In the long run, the respective probabilities that the repairman is busy in the repair of unit- 1 and unit- 2 from total failure are given by:

$$
\begin{aligned}
& B_{0}^{r_{1}}=\lim _{t \rightarrow \infty} B_{0}^{r_{1}}(t)=\lim _{h \rightarrow 1}(1-h) \frac{N_{4}(h)}{D_{2}(h)} \\
& B_{0}^{r_{2}}=\lim _{t \rightarrow \infty} B_{0}^{r_{2}}(t)=\lim _{h \rightarrow 1}(1-h) \frac{N_{5}(h)}{D_{2}(h)}
\end{aligned}
$$

But $D_{2}(h)$ at $h=1$ is zero, hence by applying L. Hospital Rule, we get 


$$
\begin{gathered}
B_{0}^{r_{1}}=-\frac{N_{4}(1)}{D_{2}^{\prime}(1)} \\
B_{0}^{r_{2}}=-\frac{N_{5}(1)}{D_{2}^{\prime}(1)}
\end{gathered}
$$

Now the expected busy period of the repairman in the repair of unit-1 and unit-2 respectively up to epoch $(t-1)$ are given by:

$$
\begin{aligned}
& \mu_{b}^{r_{1}}(t)=\sum_{x=0}^{t-1} B_{0}^{r_{1}}(x) \\
& \mu_{b}^{r_{2}}(t)=\sum_{x=0}^{t-1} B_{0}^{r_{2}}(x)
\end{aligned}
$$

So that

$$
\begin{gathered}
\mu_{b}^{r_{1} *}(h)=\frac{B_{0}^{r_{1} *}(h)}{(1-h)} \\
\mu_{b}^{r_{1}^{\prime *}}(h)=\frac{B_{0}^{r_{1}^{*} *}(h)}{(1-h)}
\end{gathered}
$$

\section{Profit Function Analysis}

We are now in the position to obtain the net expected profit incurred up to epoch $(t-1)$ by considering the characteristics obtained in earlier sections.

Let us consider,

$K_{0}=$ revenue per-unit time by the system when it is operative.

$K_{1}=$ cost per-unit time when repairman is busy in the PM of unit-1.

$K_{2}=$ cost per-unit time when repairman is busy in the repair of unit-1.

$K_{3}=$ cost per-unit time when repairman is busy in the repair of unit-2.

Then, the net expected profit incurred up to epoch $(t-1)$ given by:

$$
P(t)=K_{0} \mu_{u p}(t)-K_{1} \mu_{b}^{p m}(t)-K_{2} \mu_{b}^{r_{1}}(t)-K_{3} \mu_{b}^{r_{2}}(t)
$$

In steady state, the expected profit per unit time incurred to the system is given by:

$$
P=\lim _{t \rightarrow \infty} \frac{P(t)}{t}
$$

$$
\begin{gathered}
=K_{0} \lim _{h \rightarrow 1}(1-h)^{2} \frac{A_{0}^{*}(h)}{(1-h)}-K_{1} \lim _{h \rightarrow 1}(1-h)^{2} \frac{B_{0}^{* p m}(h)}{(1-h)} \\
-K_{2} \lim _{h \rightarrow 1}(1-h)^{2} \frac{B_{0}^{* r_{1}}(h)}{(1-h)}-K_{3} \lim _{h \rightarrow 1}(1-h)^{2} \frac{B_{0}^{* r_{2}}(h)}{(1-h)} \\
=K_{0} A_{0}-K_{1} B_{0}^{p m}-K_{2} B_{0}^{r_{1}}-K_{3} B_{0}^{r_{2}}
\end{gathered}
$$

\section{Graphical Presentation}

Consider the two non-identical unit cold standby system with the following values of parameters:

$$
\begin{array}{lccc}
a=0.1, & \alpha_{1}=0.15, & \beta_{1}=0.2, & \beta_{2}=0.4 \\
d=0.6, & b=0.9, & \pi_{1}=0.8, & \pi_{2}=0.6 \\
\alpha_{2}=0.3, & \theta_{1}=0.85, & \theta_{2}=0.7, & c=0.4 \\
\psi_{2}=0.6, & \sigma_{2}=0.4, & \lambda_{2}=0.9, & \gamma_{2}=0.1
\end{array}
$$

and $\gamma_{1}=1-\lambda_{1}, \sigma_{1}=1-\psi_{1}, K_{0}=460, K_{1}=250, K_{2}=100$, $K_{3}=50$

On the basis of the numerical values taken as: $\psi_{1}=0.01$, $\lambda_{1}=0.95$

The values of various measures of system effectiveness are obtained as:

- Mean time to system failure $(\mathrm{MTTF})=7.47$

- Steady state availability $=0.994$

- Probability that the repairman is busy in PM of unit-1= 0.0854

- Probability that the repairman is busy in the repair of unit1 from total failure $=0.317$

- Probability that the repairman is busy in the repair of unit2 from total failure $=0.0733$

- Expected profit function $=400.86$

Fig. 2 depicts the variations in MTTF with respect to the failure rate $\lambda_{1}$. We observe that the MTTF decreases uniformly as the value of $\lambda_{1}$ increases.

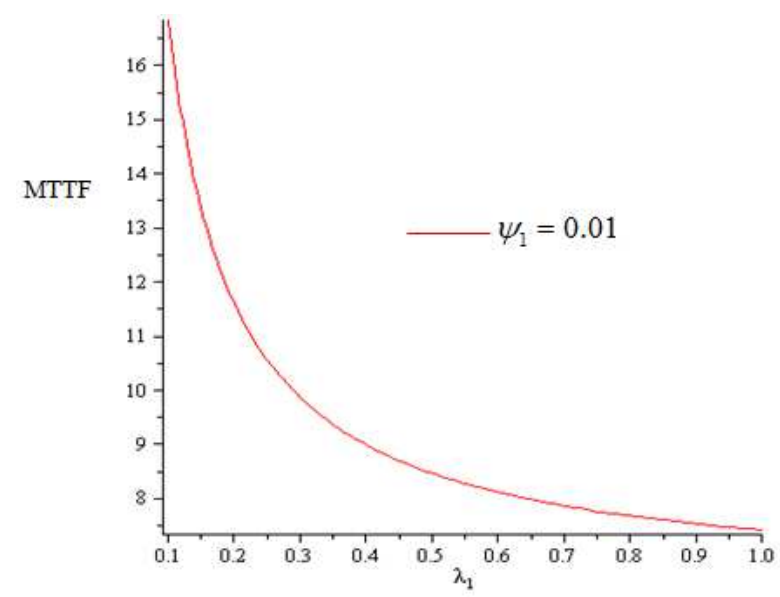

Figure 2. MTTF versus failure rate $\lambda_{1}$ 
Fig. 3 depicts the variations in MTTF with respect to the repair rate $\psi_{1}$. We observe that the MTTF increases uniformly as the value of $\psi_{1}$ increases.

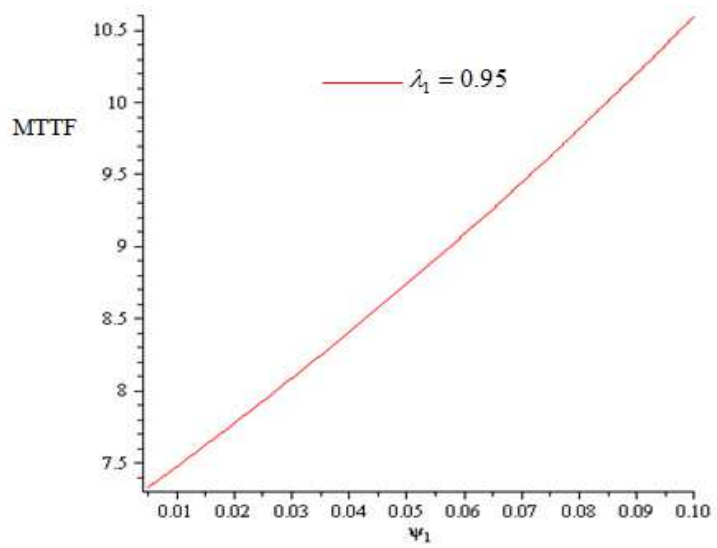

Figure 3. MTTF versus repair rate $\psi_{1}$

Fig. 4 depicts the variations in the expected profit function $P$ with respect to the failure rate $\lambda_{1}$. We observe that $P$ decreases uniformly as the value of $\lambda_{1}$ increases.

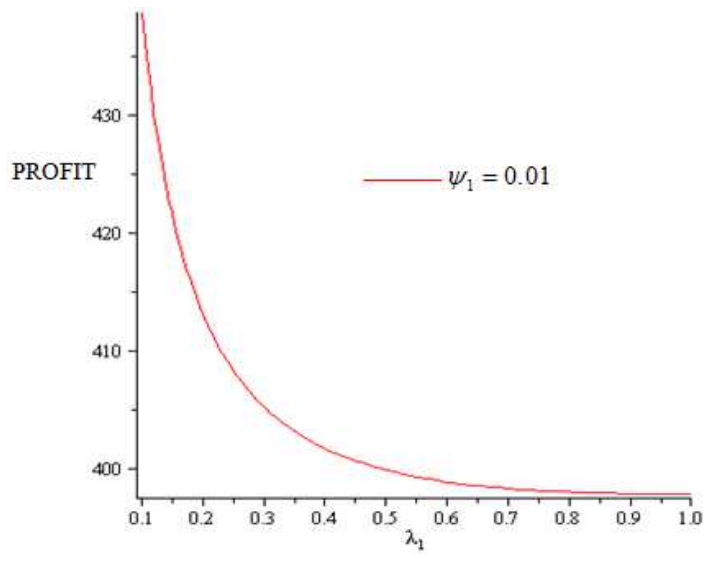

Figure 4. Profit function $P$ versus failure rate $\lambda_{1}$

Fig. 5 depicts the variations in the expected profit function $P$ with respect to the repair rate $\psi_{1}$. We observe that $P$ increases uniformly as the value of $\psi_{1}$ increases.

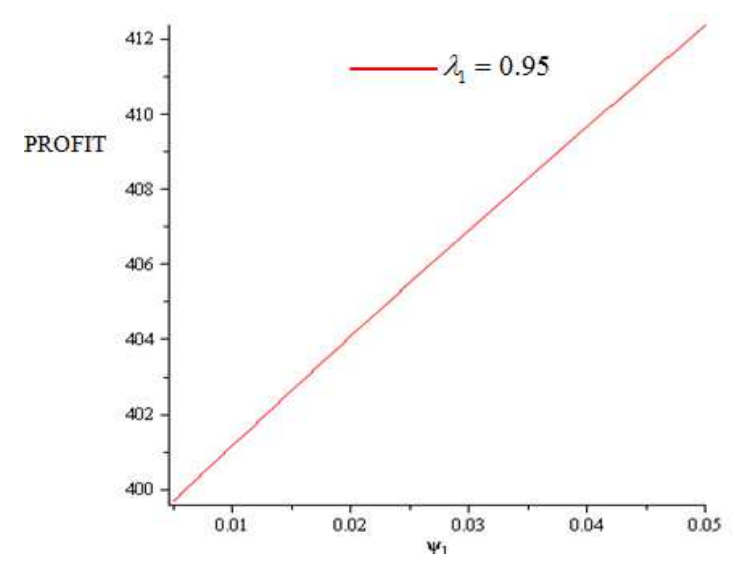

Figure 5. Profit function P versus repair rate $\psi_{1}$

\section{Conclusion}

In this paper we analyzed a two non-identical unit cold standby redundant system using semi-Markov process under discrete parametric Markov-Chain assuming three different modes of each unit(normal $(\mathrm{N})$ mode, partial failure $(\mathrm{P})$ mode, total failure $(\mathrm{F})$ mode) using regenerative point technique. Preventive maintenance was provided to the operative unit in order to increase the life time of the system. The kernel matrix and expressions for mean sojourn times, availability function, reliability function, steady-state availability, MTTF, busy period of repair man due to preventive maintenance of unit-1, busy period of repairman due to repair of unit-1 and unit-2 from total failure and expected profit function were presented assuming that the failure and repair times of a unit and time to PM and PM time are taken as discrete random variables having geometric distributions with different parameters. Numerical solutions were obtained for reliability measures of the system by the aid of Maple program at constant values of the parameters. The curves for MTSF and profit function have been drawn for different values of total failure rate and repair rate from total failure of unit-1. By comparing MTTF and expected profit $\mathrm{P}$ function versus total failure rate $\lambda_{1}$ and repair rate $\psi_{1}$, we can draw a conclusion that the MTTF and the expected profit function P decreases uniformly as the value of $\lambda_{1}$ increases, and the MTTF and the expected profit function $\mathrm{P}$ increases uniformly as the value of $\psi_{1}$ increases.

\section{Acknowledgements}

We would like to express our thanks to the Editor, and referees for their valuable and constructive comments that led to considerable improvement of the paper.

\section{References}

[1] Smith, W. L.: Regenerative Stochastic Processes. Proc. Roy. Soc (GB). Series A, pp. 232:6-31, 1955.

[2] Limnios, N., Oprisan, G.: Semi-Markov Processes and Reliability.Boston. Birkhauser, 2001.

[3] Limnios, N., Barbu, V. S.: Semi-Markov chains and hidden semi-Markov models toward applications: their use in reliability and DNA analysis. Lecture Notes in Statistics. New York. Vol. 191, 2008.

[4] Grabski, F.: Semi-Markov models of reliability and operation. Warsaw: IBS PAN (in Polish), 2002.

[5] Grabski, F.: Application of semi-Markov processes in reliability. Electronic Journal. Reliability: Theory \& Applications. Vol. 2, pp. 60-75, 2007.

[6] Grabski, F., Kołowrocki, K.: Asymptotic Reliability of Multistate System with semi-Markov states of components. Proc. of the European Conference on Safety and Reliability. Safety and Reliability. Pp. 317-322, 1999.

[7] Grabski, F.: The reliability of an object with semi-Markov failure rate. Applied Mathematics and Computation. Vol. 135, pp. 1-16, 2003. 
[8] Limnios, N., Ouhbi, B.: Nonparametric estimation of some important indicators in reliability for semi-Markov processes.Statistical Methodology.Vol. 3, pp. 341-350, 2006.

[9] Korolyuk, V.S., Turbin, A.F.: Semi-Markov processes and their applications. Naukova Dumka (in Russian), 1976.

[10] Limnios, N., Barbu, V.: Nonparametric estimation for discrete time semi-Markov processes with applications in reliability. Journal of Nonparametric Statististics. Vol. 18, pp. 483-498, 2006.

[11] Gupta, R., Bhardwaj, P.: A Discrete Parametric Markov-Chain Model of a Two Non-Identical Unit Cold Standby System with Preventive-Maintenance. International Journal of Research and Reviews in Applied Sciences. Vol. 17, pp. 2953052013.

[12] El-Said, K. M., El-Sherbeny, M. S.: Profit Analysis of a TwoUnit Cold Standby System with Preventive Maintenance and Random Change in Units. Journal of Mathematics and Statistics. Vol.1, pp. 71-77, 2005.

[13] El-Damcese, M. A., Helmy, A. N.: Study of Reliability with Mixed Standby Components. Applications and Applied Mathematics: An International Journal (AAM). Vol.7, pp. 672690, 2012.

[14] Mokaddis , G. S., Matta, C. H.: Cost Analysis of a Two Dissimilar-Unit Cold Standby Redundant System Subject To Inspection and Random Change in Units. Journal of Mathematics and Statistics. Vol. 6, pp. 306-315, 2010.

[15] Gupta, R., Bhardwaj, P.: A Two-Unit Standby System with Regular Repairman and Waiting Time of Skilled Repairman. Journal of Mathematical and Computational Science. Vol. 3, pp. 1115-1130, 2013.

[16] Gupta, R., Mumtaz, S. Z.: Stochastic Analysis of a Two-Unit Cold Standby System with Maximum Repair Time and Correlated Failures and Repairs. Journal of Quality in Maintenance Engineering. Vol. 2, pp. 66-76, 1996.

[17] Singh, S. K., Srinivasu, B.: Stochastic Analysis of a Two-Unit Cold Standby System with Preparation Time for Repair. Microelectronics Reliability. Vol. 27, pp. 55-60, 1987.
[18] GoelL. R. and Srivastva, P.: A Two-Unit Cold Standby System with Three Modes and Correlated Failures and Repairs. Microelectronics Reliability. Vol. 31, pp. 835-840, 1991.

[19] Malik, A.: Probabilistic Analysis of a Two-Unit Cold Standby System with Partial Failure. Ordinary and Expert Repairman. International Journal of Innovative Research and Studies. Vol. 13, pp. 195-209, 2014.

[20] El-Said, K. M., Abd El-Hamid, R.: Comparison of Reliability Characteristics of Two Systems with Preventive Maintenance and Different Modes. Information and Management Sciences. Vol. 19, pp. 107-118, 2008.

[21] Gupta, R., Bhardwaj, P.: A Two-Unit Standby System with Two Operative Modes of the Units and Preparation Time for Repair. Journal of Reliability and Statistical Studies. Vol. 6, pp. 87-100, 2013.

[22] Goel, L.R., Gupta, P.: Stochastic Analysis of a Deteriorating Standby System with Three Modes and Inspection. Microelectronics Reliability. Vol. 25, pp. 285-290, 1985.

[23] Goel, L.R., Gupta, R.: Analysis of a Two-Unit Cold Standby System with Three Modes and Imperfect Switching Device. Microelectronics Reliability. Vol. 24, pp. 425-429, 1984.

[24] Haggag, M. Y.: Cost Analysis of Two-Dissimilar-Unit Cold Standby System With Three States and Preventive Maintenance Using Linear First Order Differential Equations. Journal of Mathematics and Statistics. Vol. 5, pp. 395-40,2009.

[25] Sharma , V., Singh , D. Availability Analysis of a Two-Unit Standby System with Delayed Replacement Under Perfect Switching, International Journal Of Scientific \& Engineering Research, Vol. 4, pp. 1405-1409,2013.

[26] Gertsbakh, I.: Reliability theory with applications to preventive maintenance. Springer. Berlin, 2000.

[27] El-Damcese, M. A., Temraz, N. S.: Semi-Markov Model of a Series-Parallel System Subject to Preventive Maintenance. Journal of Statistics Applications and Probability. Vol.2, pp. 307-318, 2013. 